\title{
A Mixed-Fidelity Approach for Design of Low-Boom Supersonic Aircraft*
}

\author{
$\mathrm{Wu} \mathrm{Li}{ }^{1}$ \\ NASA Langley Research Center, Hampton, Virginia 23681, USA \\ Elwood Shields ${ }^{2}$ \\ Alliant Techsystems, Inc., Hampton, Virginia 23681, USA \\ Karl Geiselhart ${ }^{3}$ \\ NASA Langley Research Center, Hampton, Virginia 23681, USA
}

This paper documents a mixed-fidelity approach for the design of low-boom supersonic aircraft as a viable approach for designing a practical low-boom supersonic configuration. A low-boom configuration that is based on low-fidelity analysis is used as the baseline. Tail lift is included to help tailor the aft portion of the ground signature. A comparison of low- and high-fidelity analysis results demonstrates the necessity of using computational fluid dynamics (CFD) analysis in a low-boom supersonic configuration design process. The fuselage shape is modified iteratively to obtain a configuration with a CFD equivalent-area distribution that matches a predetermined low-boom target distribution. The mixed-fidelity approach can easily refine the low-fidelity low-boom baseline into a low-boom configuration with the use of CFD equivalent-area analysis. The ground signature of the final configuration is calculated by using a state-of-the-art CFD-based boom analysis method that generates accurate midfield pressure distributions for propagation to the ground with ray tracing. The ground signature that is propagated from a midfield pressure distribution has a shaped ramp front, which is similar to the ground signature that is propagated from the CFD equivalent-area distribution. This result confirms the validity of the low-boom supersonic configuration design by matching a low-boom equivalent-area target, which is easier to accomplish than matching a low-boom midfield pressure target.

\footnotetext{
* AIAA Paper 2010-0845, 48th AIAA Aerospace Sciences Meeting, January 2010, Orlando, Florida.

${ }^{1}$ Senior Research Engineer, Aeronautics Systems Analysis Branch, Mail Stop 442.

${ }^{2}$ Senior System Engineer, Aeronautics Systems Analysis Branch, Space Division.

${ }^{3}$ Aerospace Engineer, Aeronautics Systems Analysis Branch, Mail Stop 442.
} 


\title{
Nomenclature
}

\author{
$A_{\mathrm{e}} \quad=\quad$ equivalent area \\ $d p / p=$ (the calculated pressure - the ambient pressure $) /($ the ambient pressure $)$
}

\section{Introduction}

Low-boom supersonic aircraft design remains one of the most challenging aircraft design problems; this problem is a truly multidisciplinary design problem that frustrates many talented aircraft designers. Although designing for cruise efficiency is not a simple task, a cruise-efficient configuration can be created by designing an aerodynamically efficient wing, optimizing the wave drag with fuselage shaping, and mitigating interference problems caused by component integration with local modifications that utilizes computational fluid dynamics (CFD) analysis. To create a low-boom aircraft, not only must some measure of aerodynamic efficiency be retained, but the shapes of the various components along with the lift characteristics must be integrated in a manner that creates an acceptable pressure signature many body lengths away from the configuration. Ideally, a multidisciplinary design optimization tool should be used to reshape a baseline configuration into a low-boom design. However, the critical technology for such a tool - an adjoint solver for determining the sensitivity of the midfield pressure distributions with respect to shape changes - is not available for a complete aircraft.

This paper documents the use of a mixed-fidelity approach for the design of low-boom supersonic aircraft with a focus on fuselage shaping to minimize the difference between a CFD equivalent area $\left(A_{\mathrm{e}}\right)$ of the configuration and a predetermined low-boom target $A_{\mathrm{e}}$.

The mixed-fidelity approach is based on two recent advances in supersonic concept design and analysis capabilities: the inverse design optimization of low-boom supersonic concepts with smoothest fuselage shape modifications [1] and the integration of automated CFD analysis in conceptual design of supersonic aircraft [2]. The first capability allows one to reshape the fuselage smoothly to obtain a configuration with an $A_{\mathrm{e}}$ distribution that matches a low-boom target. Because multiple iterations are required to match the total $A_{\mathrm{e}}$ distribution of a supersonic concept with a low-boom target $A_{\mathrm{e}}$ distribution, all of the analyses involved in the previous inverse design cases are low-fidelity methods (i.e., codes that finish calculations in seconds). See reference [1] for more detailed descriptions and additional references for these low-fidelity methods. Automated CFD analysis allows a low-boom concept that has been designed by using low-fidelity analyses to be refined by revealing the additional characteristics in the $A_{\mathrm{e}}$ distribution that could not be detected by the low-fidelity analyses. The mixed-fidelity approach integrates the CFD $A_{\mathrm{e}}$ analysis into the fuselage shaping process that is documented in reference [1] to refine the low-fidelity design so that the CFD $A_{\mathrm{e}}$ distribution of the refined design is closer to a low-boom target $A_{\mathrm{e}}$. Several refinement steps may be necessary to obtain a configuration with a CFD $A_{\mathrm{e}}$ distribution that is as close to the low-boom target $A_{\mathrm{e}}$ as possible. The mixed-fidelity low-boom design method has been implemented in ModelCenter [3], which enables a conceptual designer to match the CFD equivalent-area distribution to the target within a few days starting from a low-fidelity low-boom baseline.

In theory, the validity of designing a low-boom configuration by matching a predetermined target is based on the far-field theory assumption that the complete aircraft configuration can be treated as an axisymmetric body of revolution in the boom analysis. (See reference [4] for a survey of sonic boom theory.) Even though the nonsymmetrical three-dimensional effects of a complete aircraft with nacelles and tails could render this assumption invalid, this mixed-fidelity method, which is based on $A_{\mathrm{e}}$ matching that is derived from the far-field low-boom theory, can be shown to yield a configuration with a 
reasonable front shaped ground signature that is propagated from a CFD pressure distribution of the configuration at three to ten body lengths below the configuration. This provides empirical evidence of the validity of low-boom design methods that use $A_{\mathrm{e}}$ matching instead of midfield pressure matching.

The CFD ground signatures of the final low-boom designs are calculated by using a CFD boom analysis method developed by Campbell et. al. [2,5], which has been implemented in the ModelCenter process. The ModelCenter CFD boom analysis can be set up by specifying a few parameters (such as cruise Mach number, flight altitude, cruise lift coefficient, location of the midfield pressure distribution, etc.). Then, the CFD boom analysis can be run in ModelCenter and the ground signature that is propagated from the CFD pressure distribution at 3 to 10 body lengths below the configuration can be obtained within 8 to 12 hours by using 48 Linux cluster processors.

Note that a predetermined low-boom target $A_{\mathrm{e}}$ might not be realizable for a feasible aircraft configuration. In particular, no documented method exists for generating a realizable target $A_{\mathrm{e}}$ with both a well-shaped front and aft portion of the ground signature. Reference [6] documents one attempt to use numerical optimization methods to generate realizable low-boom $A_{\mathrm{e}}$ distributions. Some preliminary results show that tail lift tailoring is an effective approach for shaping the aft portion of the ground signature of a low-boom configuration. Therefore, the tail lift is also used here to help tailor the aft portion of the ground signature. However, the development of a low-boom configuration that has both front and aft shaped portions of the ground signature propagated from the midfield pressure distribution is beyond the scope of this paper.

The remainder of the paper is organized as follows. Section II includes the details of the mixed-fidelity fuselage shaping process for low-boom design. In section III, the mixed-fidelity low-boom fuselage shaping process is demonstrated with a supersonic business jet design case. The verification results of the mixed-fidelity designs are given in section IV. The final section contains the concluding remarks.

\section{Mixed-Fidelity Low-Boom Fuselage Shaping Method}

The mixed-fidelity design process is a refinement of the low-fidelity low-boom design process, which is described in reference [1], by including automated CFD analysis. The details for the automated CFD analysis process can be found in reference [2]. The manual script execution portion of the automated CFD analysis process that is described in reference [2] also has been integrated in ModelCenter; an automated USM3D [7,8] can be run within ModelCenter for either aero analysis or boom analysis (by using SSGRID [4], which is a grid stretching code for high grid resolution up to ten body lengths below the configuration). The process is completely automated and controlled by only a few user input parameters. The process starts from the conceptual geometry of a complete supersonic aircraft, converts the aircraft geometry to a watertight CFD geometry in VGRID [9,10] format, generates sources for grid generation based on a few intuitive control parameters, uses VGRID for unstructured grid generation, uses SSGRID to shear and stretch the volume grid for improved boom prediction, and runs USM3D to obtain a CFD Euler solution. Figure 1 shows the ModelCenter process for the integrated CFD analysis. PCBOOM [11], a boom analysis code that uses ray tracing, is also integrated in ModelCenter to propagate the off-body pressure distributions to the ground. This provides a seamless process for using either CFD $A_{\mathrm{e}}$ analysis or CFD boom prediction for a supersonic concept with a turnaround time of 2 or 12 hours, respectively, with the use of 48 Linux cluster processors. The average unstructured grid sizes for CFD $A_{\mathrm{e}}$ analysis and CFD boom prediction are approximately 6 million and 15 million cells, respectively. 


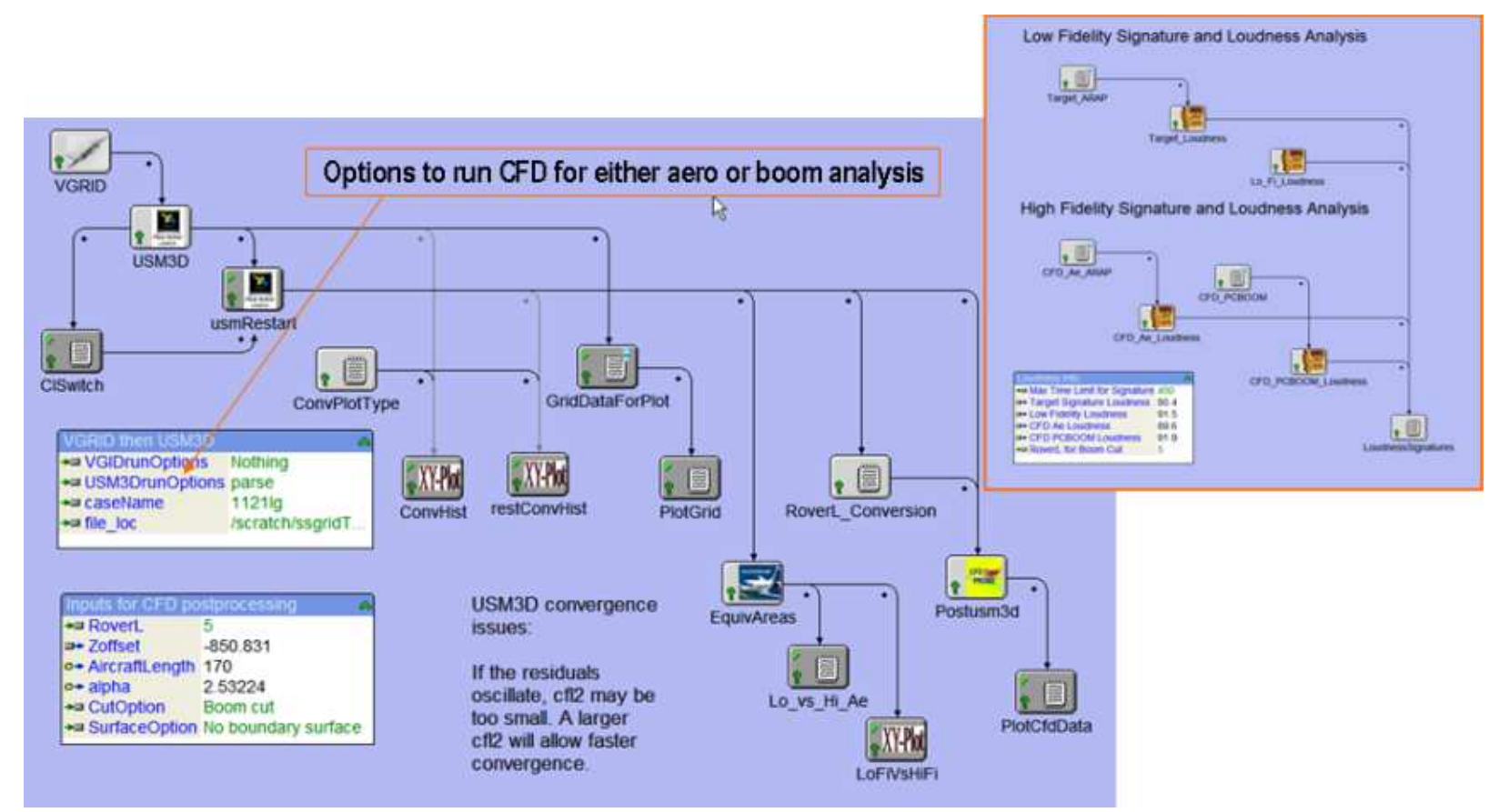

Figure 1. ModelCenter process for integrated CFD and boom analyses.

The automated CFD analysis process enables the use of CFD $A_{\mathrm{e}}$ distribution during the low-boom fuselage shaping process instead of the $A_{\mathrm{e}}$ distribution that is calculated with the use of low-fidelity methods. However, using CFD $A_{\mathrm{e}}$ analysis to calculate the $A_{\mathrm{e}}$ distribution each time the fuselage shape is changed is too time consuming. Therefore, the following mixed-fidelity $A_{\mathrm{e}}$ is used for fuselage shaping.

$$
A_{e}^{\text {mixed }}=A_{e, C F D}^{\text {total }}-A_{e, \text { old }}^{\text {fuse }}+A_{e, \text { new }}^{\text {fuse }}
$$

In the above formula, the equivalent areas for fuselage $A_{\mathrm{e}, \text { old }}$ and $A_{\mathrm{e}, \text { new }}$ are calculated by using the low-fidelity analysis that is documented in reference [1]. That is, the fuselage $A_{\mathrm{e}}$ distributions are calculated by using the fuselage as a standalone component without consideration for the volume difference that results from the intersection between the fuselage and other components of the configuration (such as the wing, the pylon, and the vertical tail). Moreover, the $A_{\mathrm{e}}$ difference that is caused by lift for the two configurations is not accounted for in the mixed-fidelity $A_{\mathrm{e}}$. In other words, the mixed-fidelity approach is based on the assumption that the (low fidelity) $A_{\mathrm{e}}$ difference between the two fuselage shapes is a reasonably accurate estimate of the actual difference in the (high fidelity) CFD $A_{\mathrm{e}}$ distributions of the two configurations.

Figure 2 shows the mixed-fidelity fuselage shaping process in ModelCenter. The goal of this process is to obtain a new fuselage shape that reduces the difference between the mixed-fidelity total $A_{\mathrm{e}}$ and the low-boom target $A_{\mathrm{e}}$ as much as possible while still retaining a reasonable configuration. This requires some judgment on the part of the designer as to how much of the target $A_{\mathrm{e}}$ should be matched. In most cases, this is determined when the front portion of the ground signature that is propagated from the mixed-fidelity total $A_{\mathrm{e}}$ distribution has been matched accurately with that of the target signature. At this point, the CFD equivalent area of the modified configuration is calculated by running the CFD analysis and comparing the results again to the target $A_{\mathrm{e}}$. If the matching result is undesirable, then another iteration of the mixed-fidelity fuselage shaping is done to further improve the matching. This process is repeated until the configuration has a reasonably shaped ground signature when the CFD equivalent area 
is used in the boom analysis. Then, the CFD analysis is run again to obtain a midfield pressure distribution that is propagated to the ground by ray tracing. This verifies whether the configuration indeed has a reasonably shaped low-boom ground signature. Figure 3 provides an overall view of the mixedfidelity low-boom configuration design process, along with the related low-fidelity design and highfidelity verification.

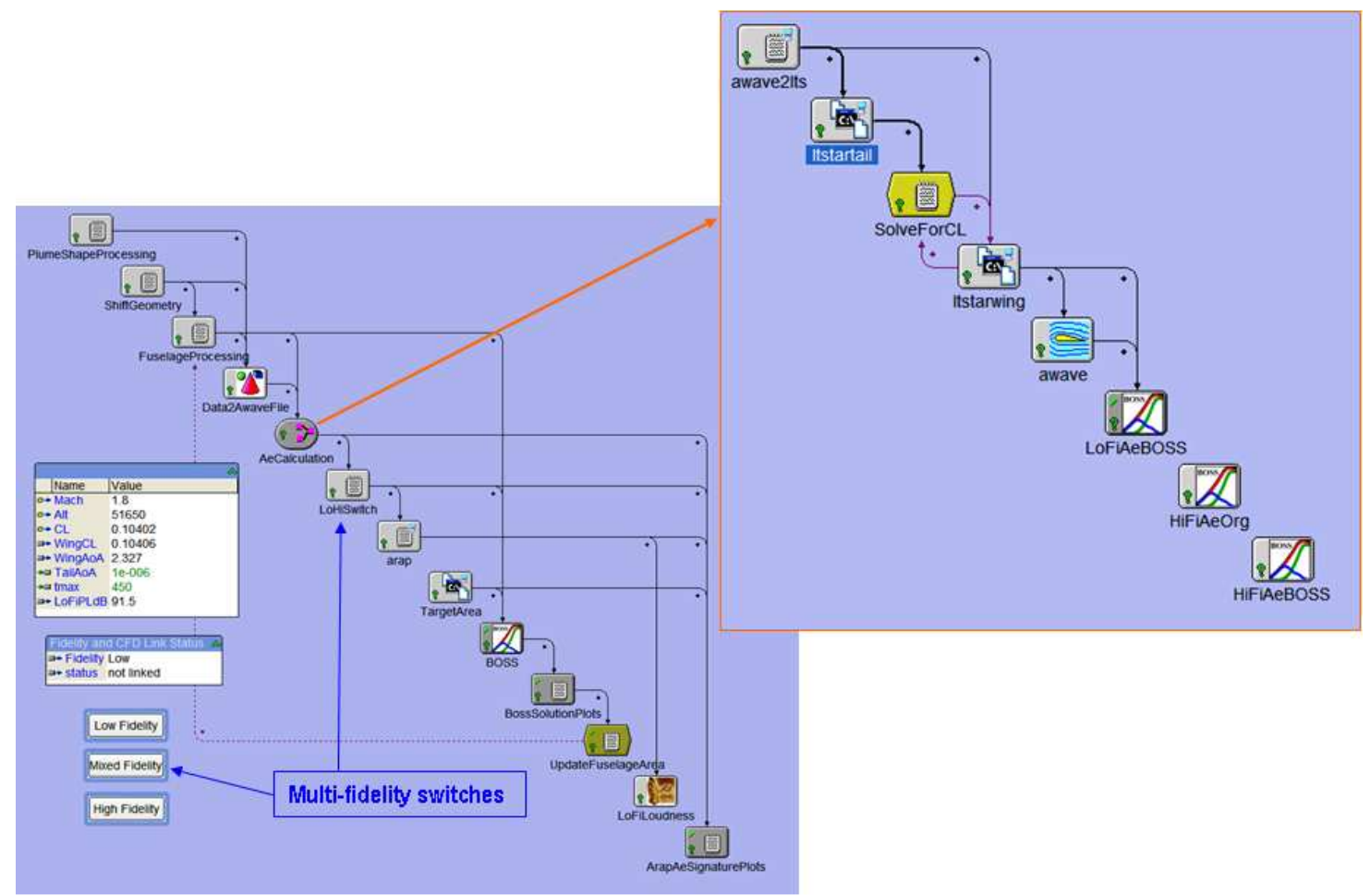

Figure 2. ModelCenter process for mixed-fidelity low-boom fuselage shaping.

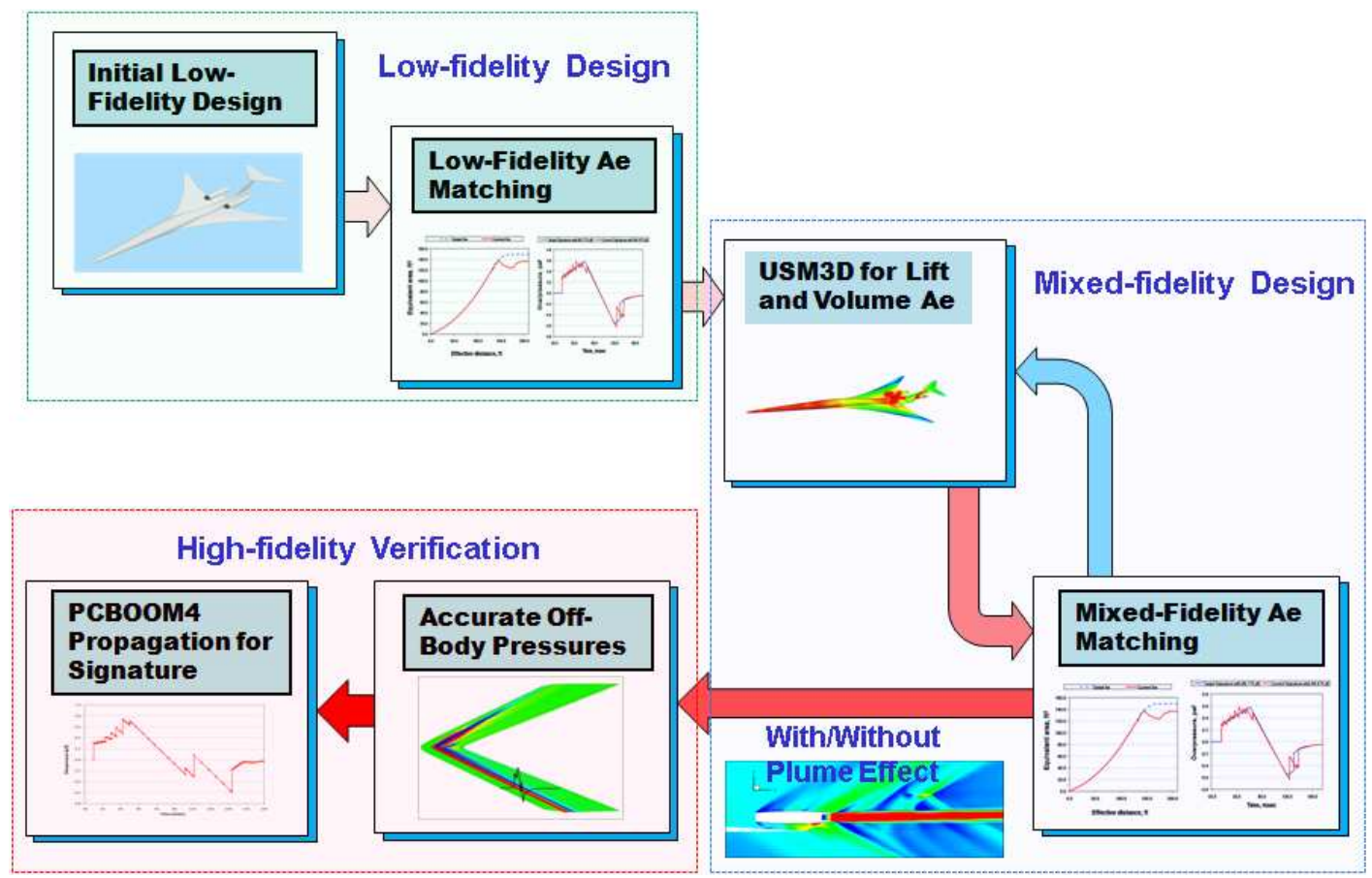

Figure 3. Flowchart of the mixed-fidelity design and analysis process. 


\section{Case Study}

A previously designed supersonic business jet configuration, shown in Figure 4, is used as a starting point to demonstrate the mixed-fidelity low-boom fuselage shaping process. The configuration is developed to achieve the best performance, expressed as maximum range, for a given takeoff gross weight of 100,000 lb and a balanced field length of $7000 \mathrm{ft}$. The cabin is to be equivalent to that of a Citation X, and the cruise Mach number is 1.8. The aircraft length is $170 \mathrm{ft}$. This configuration is designed at the conceptual level by using low-fidelity analysis codes to satisfy all of the practical considerations of the various disciplines, such as aerodynamics, structures, systems, low-speed performance, stability and control, and landing-gear placement. The details for the low-fidelity low-boom design process can be found in reference [1].

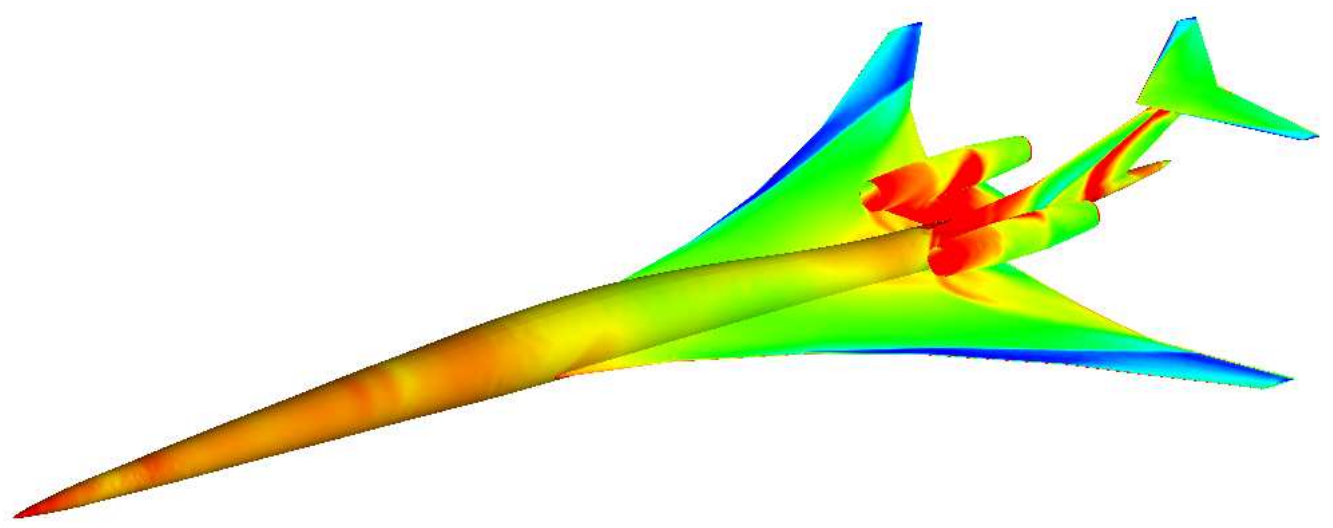

Figure 4. Baseline supersonic concept.

The baseline was designed to match as much of the target $A_{\mathrm{e}}$ as possible while still maintaining a reasonable configuration. This was possible up to an effective distance of approximately $145 \mathrm{ft}$ and resulted in a good match of the forward portion of the ground signature (see Figure 5). Tail lift was then used to help tailor the aft portion of the ground signature. An incidence of $2.5 \mathrm{deg}$ was added to the horizontal tail; this changed the aft portion of the signature so that it was no longer an $\mathrm{N}$-wave. At this point, additional changes to the configuration that could potentially improve the matching of the target $A_{\mathrm{e}}$ were considered and found to be unreasonable or to have an adverse impact on the configuration's performance. The complete low-fidelity low-boom design process resulted in a significant decrease in the perceived loudness of the baseline configuration from 91.8 PLdB to 84.5 PLdB. The low-fidelity $A_{\mathrm{e}}$ matching and ground signature analysis results of the baseline are shown in Figure 5.
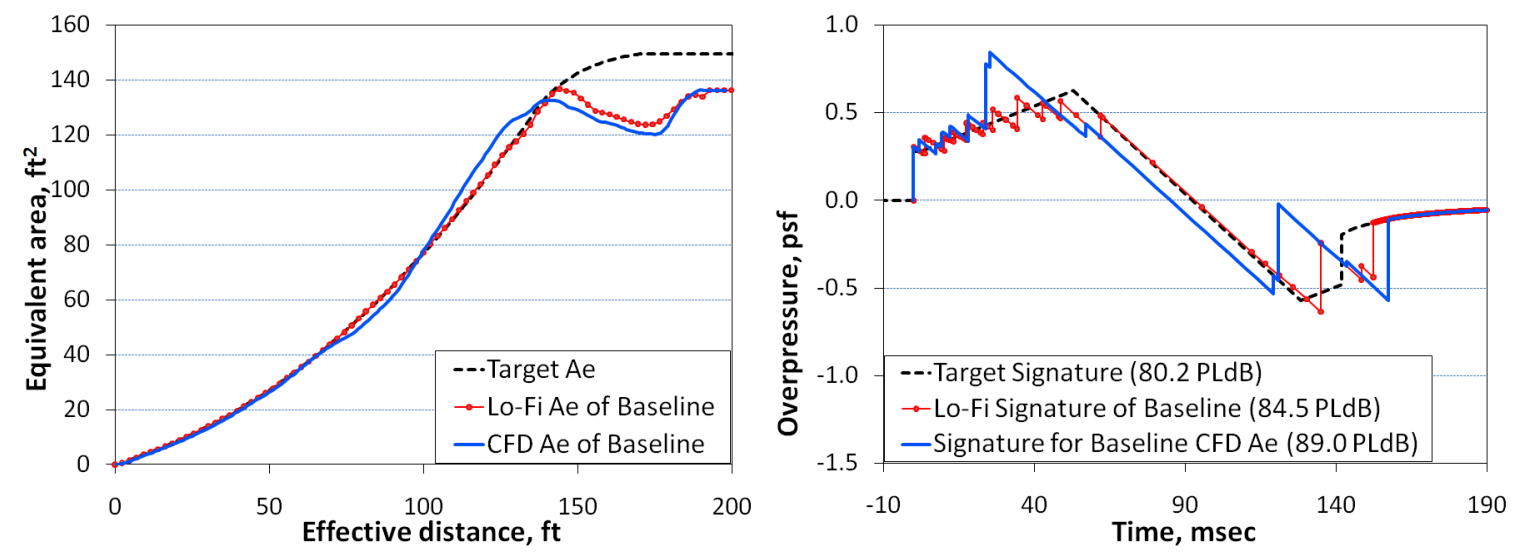

Figure 5. $A_{\mathrm{e}}$ and ground signature analysis of the baseline. 
Next, a CFD analysis is run. A comparison of the CFD $A_{\mathrm{e}}$ distribution of the baseline with the lowfidelity $A_{\mathrm{e}}$ distribution shows significant differences (see the left plot in Figure 5). The front portion of the ground signature from the CFD $A_{\mathrm{e}}$ distribution exhibits a significant spike, which is approximately a 40 percent increase in the maximum overpressure over that of the low-fidelity ground signature, and a pronounced change to the aft portion of the signature (see the right plot in Figure 5). These discrepancies demonstrate the value of using CFD analysis in the low-boom supersonic configuration design.
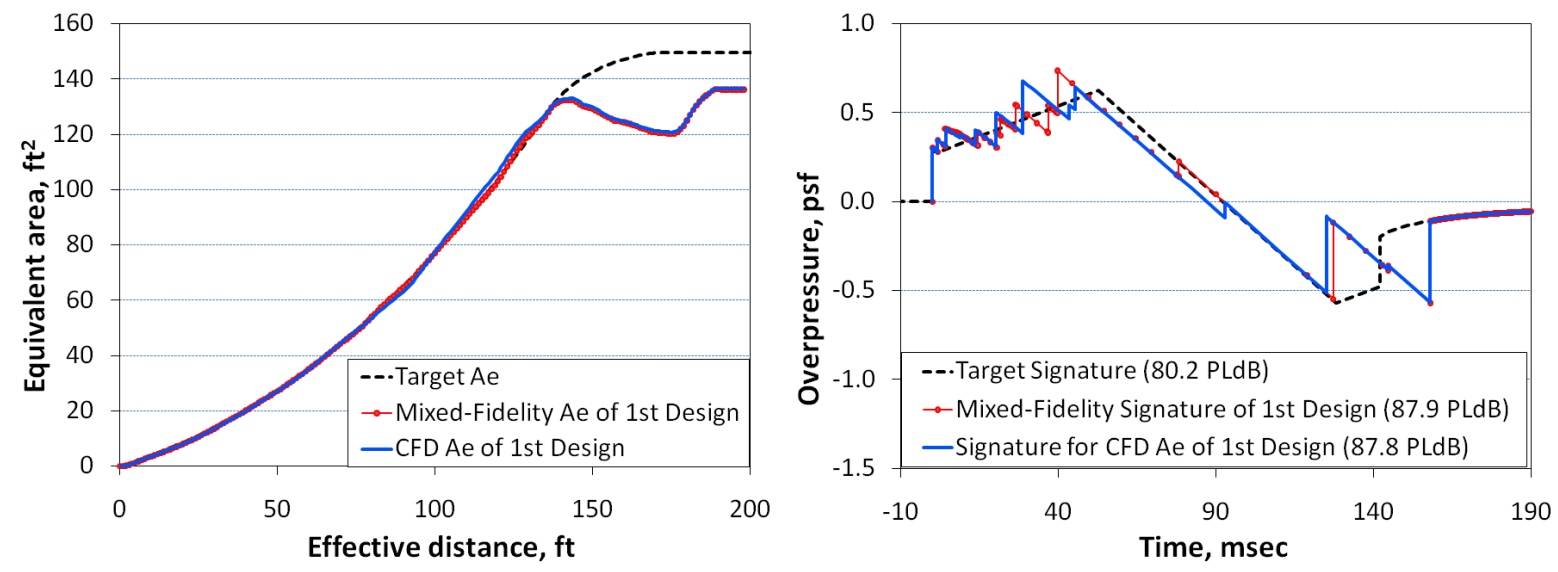

Figure 6. $A_{\mathrm{e}}$ and signature analysis of the $\mathbf{1}^{\text {st }}$ mixed-fidelity design.
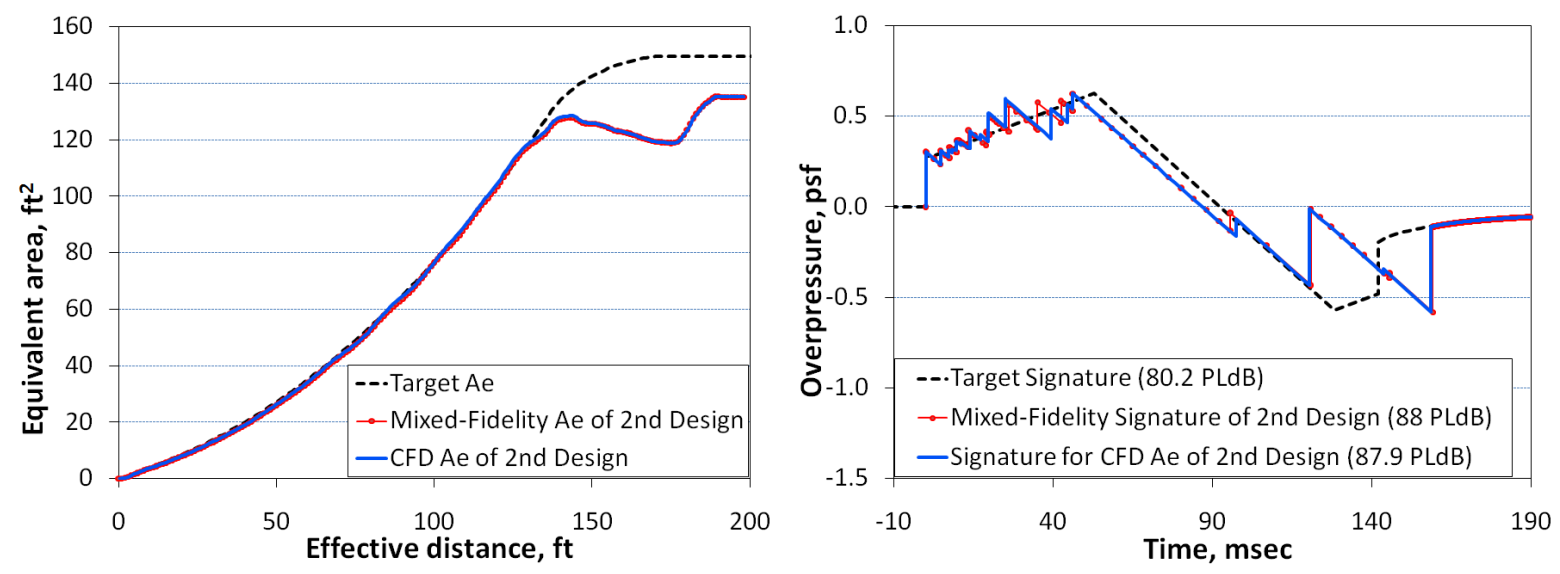

Figure 7. $A_{\mathrm{e}}$ and signature analysis of the $2^{\text {nd }}$ mixed-fidelity design.
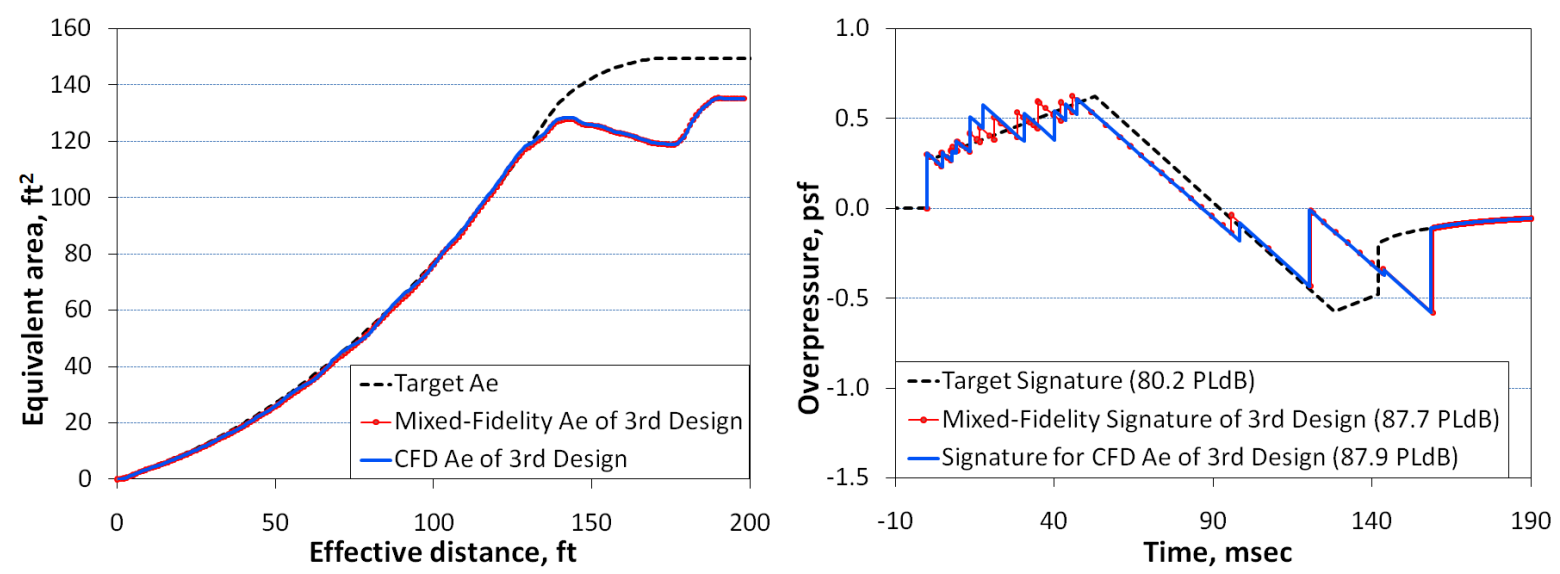

Figure 8. $\quad A_{\mathrm{e}}$ and signature analysis of the $3^{\text {rd }}$ mixed-fidelity design. 
Three iterations of mixed-fidelity fuselage shaping were applied to the baseline to obtain a configuration for which the CFD $A_{\mathrm{e}}$ matched the target $A_{\mathrm{e}}$ and for which a reasonable ground signature was maintained. The analysis results are shown in Figures 6-8.

In each of the mixed-fidelity iterations, the equivalent area from the CFD analysis that is attributable to lift remains the same, and the total mixed-fidelity $A_{\mathrm{e}}$ changes only because of changes in the fuselage volume distribution, as detailed in section II. The changes to the fuselage volume are accomplished by using the smoothing and fitting tools, such as BOSS [1] that are integrated in ModelCenter. Each iteration is completed when the match between the mixed-fidelity $A_{\mathrm{e}}$ and the target $A_{\mathrm{e}}$ is close enough to create a reasonable ground signature that is propagated from the mixed-fidelity $A_{\mathrm{e}}$ while retaining a reasonable configuration. Initially, because of relatively large differences between the CFD $A_{\mathrm{e}}$ and target $A_{\mathrm{e}}$ of the baseline, the first mixed-fidelity iteration requires appreciable changes to the fuselage in order for the mixed-fidelity $A_{\mathrm{e}}$ to effectively match the target $A_{\mathrm{e}}$ and result in a reasonable ground signature (see Figure 6). At this point, a second CDF analysis is completed, and a more refined equivalent area from the CFD analysis is calculated. With this result, only small changes to the fuselage are necessary to create an acceptable match between the mixed-fidelity $A_{\mathrm{e}}$ and the target $A_{\mathrm{e}}$; the reshaped design has a slightly better ground signature (see Figure 7). The iterative process is necessary because once the fuselage is changed in the first matching attempt, the lift and volume are no longer exactly represented by the CFD analysis results. For the mixed-fidelity approach, when the differences between the CFD $A_{\mathrm{e}}$ and the target $A_{\mathrm{e}}$ are small, any further reduction between the mixed-fidelity $A_{\mathrm{e}}$ and the target $A_{\mathrm{e}}$ may not result in a reduction of the difference between the CFD $A_{\mathrm{e}}$ and the target $A_{\mathrm{e}}$ for a new mixed-fidelity design (see the third iteration of the mixed-fidelity design in Figure 8).

\section{Verification of Mixed-Fidelity Low-Boom Design}

The low-boom configurations that are designed by using $A_{\mathrm{e}}$ analysis must be verified by analyzing the ground signatures that are propagated from the midfield pressure distributions. The USM3D solution with a stretched grid is used to generate a high-resolution midfield pressure distribution for the boom analysis. The pressure distribution at three body lengths below the configuration is used as input for the PCBOOM analysis. See Figure 9 for the target $d p / p$ and $d p / p$ for the mixed-fidelity designs at three body lengths below the configuration $(H / L=3)$.

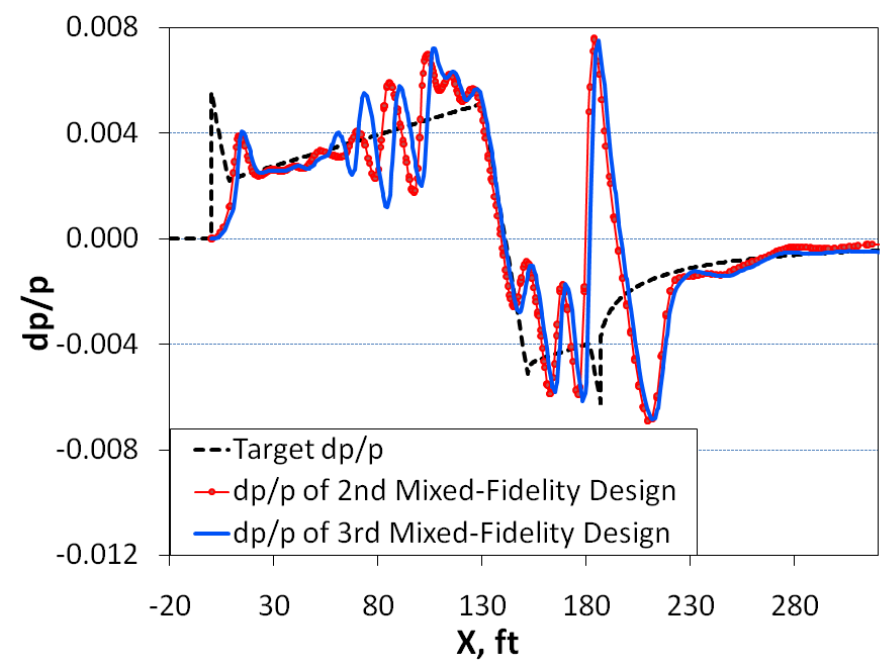

Figure 9. Comparison of $d p / p$ at $H / L=3$. 

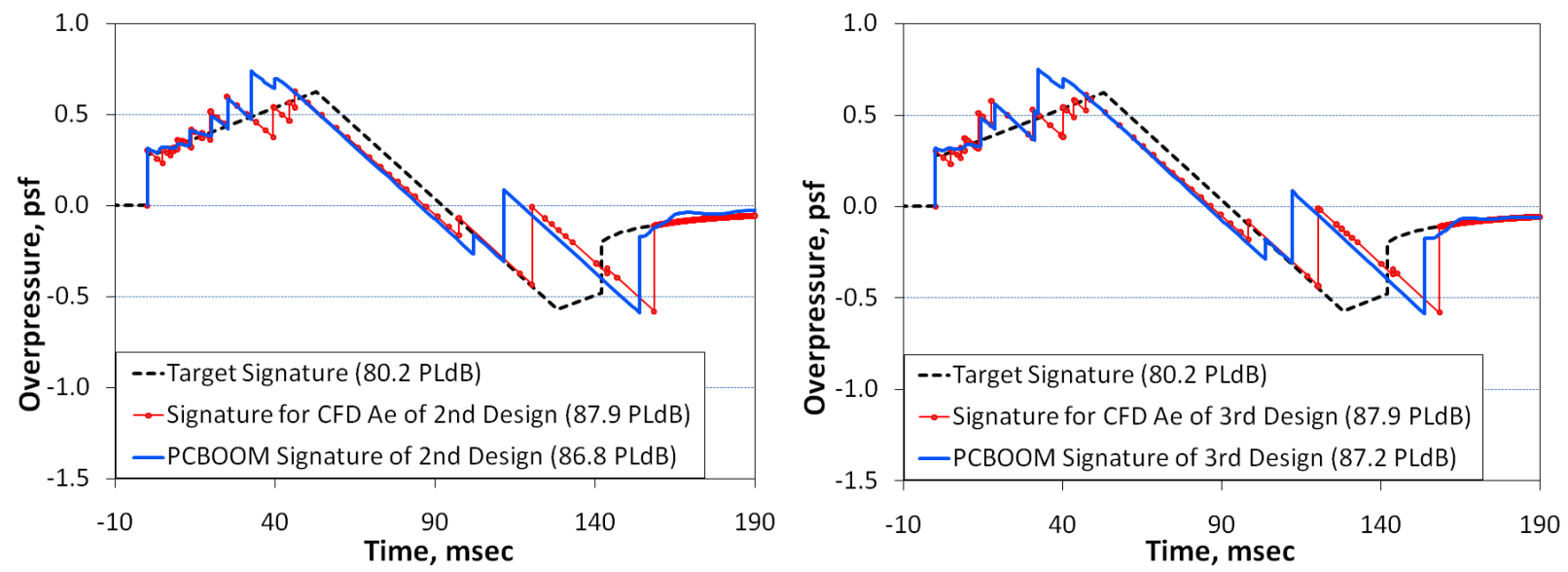

Figure 10. PCBOOM ground signatures propagated from $d p / p$ at $H / L=3$.

The ground signatures for the second and third mixed-fidelity designs are shown in Figure 10. Even though the signature that is propagated from CFD $A_{\mathrm{e}}$ differs from the signature that is propagated from the midfield pressure distribution, both front shapes are similar to the ramp target signature. At the moment, the difference between the CFD $A_{\mathrm{e}}$ and the midfield pressure distribution signatures does not significantly affect the perceived loudness, but such differences could adversely impact a both front and aft shaped ground signature. From Figure 9, we know that modifications to the configuration are necessary to cause $d p / p$ to match the target $d p / p$ at three body lengths below the configuration.

\section{Concluding Remarks}

A low-fidelity low-boom design process has been enhanced with the use of automated computational fluid dynamics (CFD) analysis. The resulting mixed-fidelity low-boom design process can be used to match a CFD $A_{\mathrm{e}}$ of the configuration to a predetermined low-boom target $A_{\mathrm{e}}$ with a few CFD aero analysis runs. A low-fidelity low-boom configuration was used as the baseline to demonstrate the mixedfidelity design capability. The final mixed-fidelity designs were verified by propagation of off-body pressure distributions that were calculated by using USM3D with stretched grids. The CFD ground signatures of the mixed-fidelity designs differed slightly from the ground signatures that were propagated from the CFD $A_{\mathrm{e}}$, but all of the signatures had front shapes that were similar to the ramp target signature. This study provides empirical evidence that CFD $A_{\mathrm{e}}$ matching is a viable method for obtaining a configuration that is close to a low-boom design with CFD boom analysis.

\section{Acknowledgment}

The authors would like to thank Jim Fenbert at Alliant Techsystems, Inc. for his contribution to the ModelCenter process development. 


\section{References}

[1] Li, W., Shields, E., and Le, D., "Interactive Inverse Design Optimization of Fuselage Shape for Low-Boom Supersonic Concepts," Journal of Aircraft, Vol. 45, No. 4, 2008, pp. 1381-1398.

[2] Li, W., Campbell, R., Geiselhart, K., Shields, E., Nayani, S., and Shenoy, R., "Integration of Engine, Plume, and CFD Analyses in Conceptual Design of Low-Boom Supersonic Aircraft,” AIAA Paper 2009-1171, January 2009.

[3] ModelCenter Ver. 6.0, Design Integration Software, Phoenix Integration, Blacksburg, VA, 2004.

[4] Plotkin, K., "Review of Sonic Boom Theory," AIAA Paper 1989-1105, April 1989.

[5] Campbell, L., Carter, M., and Deere, K., and Waithe, K., "Efficient Unstructured Grid Adaptation Methods for Sonic Boom Prediction," AIAA Paper 2008-7327, August 2008.

[6] Plotkin, K., Rallabhandi, S., and Li, W., "Generalized Formulation and Extension of Sonic Boom Minimization Theory for Front and Aft Shaping," AIAA Paper 2009-1052, January 2009.

[7] Frink, N., Pirzadeh, S., Parikh, P., Pandya, M., and Bhat, M., "The NASA Tetrahedral Unstructured Software System," The Aeronautical Journal, Vol. 104, No. 1040, 2000, pp. 491-499.

[8] USM3D Users Manual Version 6.0, http://tetruss.larc.nasa.gov/usm3d/, accessed on August 18, 2008.

[9] Pirzadeh, S., "Three-Dimensional Unstructured Viscous Grids by the Advancing-Layers Method," AIAA Journal, Vol. 34, No. 1, 1996, pp. 43-49.

[10] VGRID Unstructured Grid Generator, http://tetruss.larc.nasa.gov/vgrid/, accessed on August 18, 2008.

[11] Plotkin, K., and Grandi, F., "Computer Models for Sonic Boom Analysis: PCBoom4, CABoom, BooMap, CORBoom," Wyle Report WR 02-11, June 2002. 